\title{
Protective effects of atorvastatin against oxidized LDL-induced downregulation of KLF expression in EA.hy926 cells
}

\author{
YAN GAO $^{1 *}$, XIAN-FENG LIU ${ }^{1 *}$, XUE-CHUN LU ${ }^{2}$, CONG MA $^{1}$, JIAN CAO $^{1}$ and LI FAN ${ }^{1}$ \\ ${ }^{1}$ First Geriatric Cardiology Division and ${ }^{2}$ Department of Geriatric Hematology, Chinese PLA \\ General Hospital, Beijing 100853, P.R. China
}

Received February 19, 2012; Accepted April 12, 2012

DOI: 10.3892/ijmm.2012.999

\begin{abstract}
Krüppel-like transcription factors (KLFs) play a key role in both vascular development and pathophysiological processes, but little is known about the relationship between KLFs and oxidized low-density lipoprotein (ox-LDL). We investigated the effects of ox-LDL on KLF expression. Furthermore, since atorvastatin is commonly used to treat high cholesterol, we also examined the role of this drug in the regulation of KLF expression. The human umbilical vein endothelial cell line EA.hy926 was treated with atorvastatin and ox-LDL alone or in combination, and KLF expression was examined by DNA microarray, semi-quantitative real-time PCR, western blot and immunofluorescence analyses. Atorvastatin upregulated KLF expression in EA.hy926 cells in both the quiescent and ox-LDL-induced inflammatory states, suggesting that KLFs were novel participants in the vascular endothelial dysfunction response to ox-LDL. Our study demonstrated that atorvastatin increased both the mRNA and protein levels of KLF in quiescent EA.hy926 cells. Moreover, atorvastatin counteracted the ox-LDL-induced downregulation of KLF expression.
\end{abstract}

\section{Introduction}

Oxidized low-density lipoprotein (ox-LDL) has been demonstrated to be a key molecule in the initiation and development of atherosclerotic plaque (1), which is abundant in atherosclerotic arterial walls and has numerous detrimental effects on endothelial cell function (2). Recent clinical research has shown the association between circulating ox-LDL and preclinical atherosclerosis, coronary and peripheral artery atherosclerosis,

Correspondence to: Dr Li Fan, First Geriatric Cardiology Division, Chinese PLA General Hospital, Fuxing Road 28, Beijing 100853, P.R. China

E-mail: fanli301@163.com

${ }^{*}$ Contributed equally

Key words: atorvastatin, Krüppel-like transcription factors, EA.hy926 endothelial cells, oxidized low-density lipoprotein and acute coronary syndromes (ACS) (3). The level of ox-LDL in circulation is a marker of the severity and course of ACS (4). Moreover, ox-LDL levels are independently associated with higher risk of progression in lacunar strokes (5). Recent studies have identified several mechanisms by which ox-LDL exerts its atherogenic effects, including activation of endothelial cell arginase II, which decreases endothelial nitric oxide (NO) production (6) and upregulation of inflammatory genes such as tumor necrosis factor (TNF)- $\alpha$ (7), adhesive factors, monocyte chemotactic agents (8) and metalloproteinases $(9,10)$. Through these mechanisms, ox-LDL facilitates endothelial dysfunction, platelet aggregation and thrombus formation, leading to augmentation of the inflammatory reaction and destabilization of the atherosclerotic plaques (11). Interestingly, low concentrations of ox-LDL appear to have an opposite effect, as in vitro studies have shown that ox-LDL at low concentration can promote angiogenesis and activate nitric oxide synthesis in human coronary artery endothelial cells (12). These contradictory data show us that the mechanism of ox-LDL activity on endothelial cells is far beyond our current understanding.

Krüppel-like factors (KLF) are a subclass of evolutionarily conserved zinc finger-containing transcription factors. The expression of KLF2 and KLF4 has been documented in endothelial cells and the overexpression of these factors induces expression of multiple anti-inflammatory and anti-thrombotic factors, including endothelial nitric oxide synthase (NOS) and thrombomodulin. KLF2 and KLF4 are also novel regulators of endothelial activation in response to pro-inflammatory stimuli (13-15); however, no studies have been performed on the role of other KLF family members in endothelial function. Because ox-LDL is one of the initial triggers of atherosclerosis, these studies led us to the question whether ox-LDL exerts its atherogenic effects by signaling through the KLF family members. KLF2 and KLF4 showed opposing effects on some factors compared with ox-LDL, including eNOS and thrombomodulin, so we hypothesized that ox-LDL might reduce KLF production in endothelial cells.

Atorvastatin is a synthetic 3-hydroxy-3-methylglutaryl coenzyme A (HMG-CoA) reductase inhibitor and is used to treat patients with high cholesterol. In general, statins regulate endothelial function, inhibit macrophage activities, modulate several inflammatory mechanisms involved in the atherosclerotic process and inhibit migration and proliferation of endothelial smooth muscle cells. Studies have shown that atorvastatin decreases the 
Table I. Primers for real-time quantitative RT-PCR.

Gene name

Primer sequence

Amplicon size (bp)

\begin{tabular}{llc}
\hline GAPDH & F: TGCGCAGAAAACAAGATGAG & 114 \\
& R: CACCTTCACCGTCCAGTTT & 140 \\
KLF2 & F: GCAAACGCACCGCCACTCACACCT & 140 \\
& R: CTTCCAGCCGCAGCCGTCCCAGTT & 134 \\
KLF3 & F: GTGATTATGATGGATGCAACAAA & \\
& R: TTCATCAGACCGAGCAAACTT & 104 \\
KLF4 & F: GCGGGCTGCGGCAAAACCTACAC & \\
& R: CATCCACAGCCGTCCCAGTCACAG & 87 \\
KLF6 & F: AGCTCCTCTGTCACCTCCAC & 118 \\
KLF7 & R: CAGCTCCCCGGGCACGCAA & 105 \\
& F: GTTTGCACGAAGCGATGAG & 105 \\
KLF9 & R: ATGTGGAGGGCAAGATGGTC & 112 \\
KLF13 & F: GAAACACGCCTCCGAAAAG \\
& R: TCACCTGTATGCACTCTGTAATGG & \\
& F: TCGGGAGAATACAGCTCCGATTTCT & \\
\hline
\end{tabular}

amount of circulating ox-LDL (16) and increases the expression of atheroprotective genes such as KLF2 (17) and its downstream targets, namely, endothelial nitric oxide synthase (eNOS) and thrombomodulin (15), so we hypothesized that atorvastatin may also modulate other KLF family members.

This study aims to investigate the effect of ox-LDL on KLF expression and the role of atorvastatin in the regulation of KLF expression with or without ox-LDL induction. To conduct our experiments, we selected EA.hy926 cells as our model of macrovascular endothelial cells.

\section{Materials and methods}

Materials. The human umbilical vein endothelial cell line EA.hy926 was purchased from the cell bank of Institute of Cellular Biology (Chinese Academy of Science, Shanghai, China). Cell culture materials were from Costar (Corning Incorporation, Corning, NY, USA). Atorvastatin was purchased from the National Institute for the Control of Pharmaceutical and Biological Products (China). Other reagents are indicated in the text.

Cell culture and reagent preparation. The EA.hy926 endothelial cells were cultured in Dulbecco's modified Eagle's medium (DMEM) with $10 \%$ fetal bovine serum (FBS) (Gibco-BRL, Gaithersburg, MD, USA) and $1 \%$ penicillin/streptomycin at $37^{\circ} \mathrm{C}$ in a humidified atmosphere containing $95 \% \mathrm{O}_{2}$ and $5 \% \mathrm{CO}_{2}$. Experiments were performed with cells grown to a confluency of $80 \%$. EA.hy926 cells at passages 3-5 were used in this study. Atorvastatin was dissolved in dimethyl sulfoxide (DMSO; Sigma, St. Louis, MO, USA) (stock $10 \mathrm{mM}$ ) and added to the cells at the indicated concentrations for the entire incubation period. The equivalent amount of DMSO was added to the control samples. The final concentration of DMSO never exceeded $0.1 \%$ and did not affect cell viability (data not shown).
DNA microarray. After EA.hy926 cells were treated with atorvastatin $(10 \mu \mathrm{M})$ or control (DMSO) for $24 \mathrm{~h}$, gene expression profiles were determined with Affymetrix U133A plus 2.0 genechip (CapitalBio Corporation, Beijing, China), which included 47,000 characterized human genes. Each group had 3 biological repeats.

Total-RNA was isolated using TRIzol ${ }^{\circledR}$ (Invitrogen, Inc., Grand Island, NY, USA) and purified using RNeasy spin columns (Qiagen, Hilden, Germany). Total-RNA (7 $\mu \mathrm{g})$ was used to synthesize cDNA by using the Superscript II doublestranded cDNA synthesis kit (Applied Biosystems, Carlsbad, CA, USA) and the cDNA was then used for in vitro transcription in the presence of biotin-labeled ribonucleotides (biotin-11CTPs und biotin-16-UTPs) to yield biotin-labeled cRNA. The biotin-labeled RNA fragments were hybridized to the probe array during $16 \mathrm{~h}$ of incubation, and then the array was stained with streptavidin-phycoerythrin conjugate and scanned by the GeneChip ${ }^{\circledR}$ Scanner 3000. Raw data were analyzed using Affymetrix ${ }^{\circledR}$ GeneChip ${ }^{\circledR}$ Operating Software Version 1.4.

Semi-quantitative real-time polymerase chain reaction $(P C R)$. RNA was extracted using TRIzol and quantified by measuring the absorbance at $260 \mathrm{~nm}$. Reverse transcription was performed using the One Step RT-PCR kit (Promega Corporation, Madison, WI, USA), according to the manufacturer's instructions. cDNA samples $(2 \mu \mathrm{l})$ were amplified in $20 \mu \mathrm{l}$ of $1 \mathrm{X}$ SYBR-Green PCR master mix (Applied Biosystems) and real-time PCR was performed on duplicate samples by using the Applied Biosystems ABI PRISM ${ }^{\circledR} 7300$ Real-Time PCR System with the following cycling parameters: initial denaturation at $95^{\circ} \mathrm{C}$ for $10 \mathrm{~min}$, followed by 40 cycles of denaturation at $95^{\circ} \mathrm{C}$ for $15 \mathrm{sec}$ and annealing/extension at $61^{\circ} \mathrm{C}$ for $31 \mathrm{sec}$. Data were normalized to human GAPDH mRNA levels as an endogenous control and were expressed relative to the DMSO-treated control using the 2- $\Delta \Delta \mathrm{Ct}$ method. The 
A
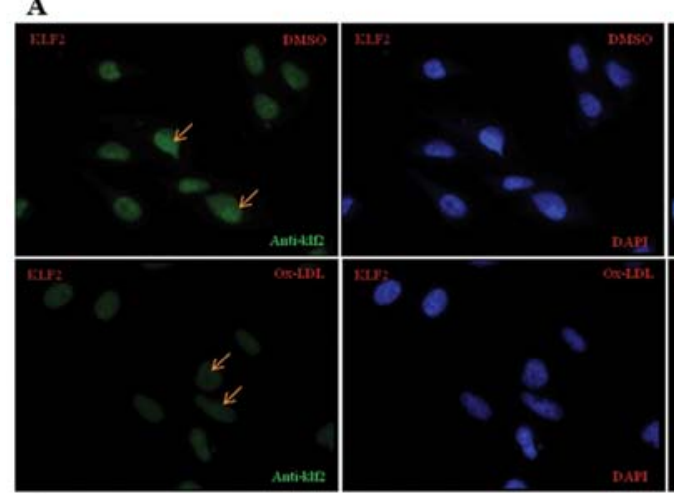

B
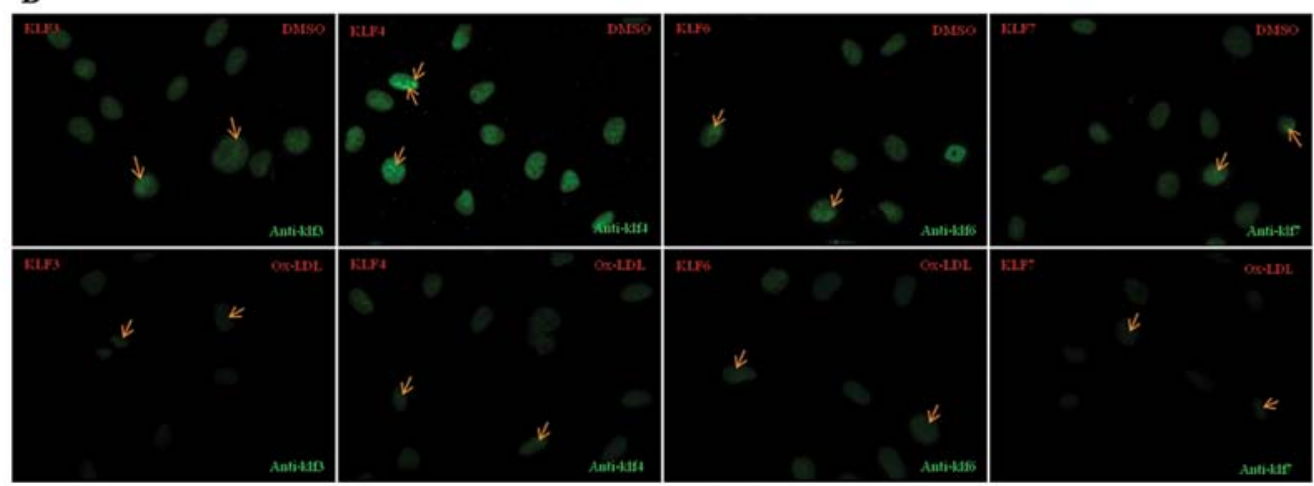

Figure 1. Effect of ox-LDL on KLF protein expression in EA.hy926 cells. (A and B) Representative immunofluorescence images are shown here. KLF expression is shown for control (DMSO) and ox-LDL $(100 \mu \mathrm{g} / \mathrm{ml})$ groups. Arrows in images indicate KLF protein expression. Fluorescence intensity was significantly reduced in ox-LDL group compared with DMSO control. Blue fluorescence represents nuclear staining using DAPI; grey fluorescence represent the merged image. (C) Bar graphs show the mean KLF fluorescent intensity values \pm SEM ( $n=3$ each). ${ }^{*} \mathrm{P}<0.05$ ox-LDL vs. DMSO group. DM, DMSO.

primers were designed using Primer Express ${ }^{\circledR}$ Primer Design Software V3.0 (Applied Biosystems) (Table I).

Western blot analysis. Following treatment, cells were washed with ice-cold phosphate-buffered saline (PBS) and lysed with NucBuster ${ }^{\mathrm{TM}}$ protein extraction kit (Calbiochem Merck, Co., Darmstadt, Germany). Cell lysates were separated on SDS-PAGE and transferred to Whatman nitrocellulose membranes. The membranes were blocked with Blotto-Tween (5\% nonfat milk and $0.05 \%$ Tween-20 in PBS) and incubated with primary antibodies against KLF2, KLF3, KLF4, KLF6 and KLF7 for $2 \mathrm{~h}$ at room temperature. After washing, the membranes were incubated with horseradish peroxidaseconjugated secondary antibodies. The bands were detected by chemiluminesence detection agents. The blots were subjected to densitometry and the bands were analyzed by Gene Genius bio imaging system (Syngene, Frederick, MD, USA).

Immunofluorescence and confocal microscopy. To explore the relationship between ox-LDL and the KLFs, we used immunofluorescence with specific primary antibodies against the members of the KLF family. EA.hy926 cells were plated onto glass coverslips, which were incubated in DMEM in the presence of DMSO control (A), $10 \mu \mathrm{M}$ atorvastatin (B), $100 \mu \mathrm{g} / \mathrm{ml}$ ox-LDL (C) or $10 \mu \mathrm{M}$ atorvastatin $+100 \mu \mathrm{g} / \mathrm{ml}$ ox-LDL (D) for $24 \mathrm{~h}$. After incubation, the cells were fixed with $4 \%$ formaldehyde for $15 \mathrm{~min}$, stained with anti-KLF antibody [goat KLF2, goat KLF3 and rabbit KLF6 antibodies (Santa Cruz Biotechnology, Inc., Santa Cruz, CA, USA), 1/500; mouse
KLF4 antibody (ProMab Biotechnologies, Inc., Richmond, CA, USA), 1:800; rabbit KLF7 antibody (Proteintech Group, Inc., Chicago, IL, USA), $1: 400]$ at $4^{\circ} \mathrm{C}$ overnight, and then incubated for $30 \mathrm{~min}$ with fluorescein-conjugated secondary antibodies (rabbit anti-goat IgG/HRP, 1:40,000; goat antimouse IgG/HRP,1:80,000 and goat anti-rabbit IgG/HRP, 1:40,000). Finally, immunostaining was performed, visualized and photographed using a Leica TCS-SP confocal laserscanning microscope $(11,12)$.

Statistical analysis. All experiments were performed in duplicate or triplicate with at least 2 biological replicates. All data are presented as mean \pm standard deviation (SD). Comparisons among groups were performed by one-way ANOVA followed by a posteriori Tukey's test. Differences were accepted as statistically significant when $\mathrm{P}<0.05$.

\section{Results}

Ox-LDL downregulates KLF expression in EA.hy926 cells. To investigate the effect of ox-LDL on KLF expression, we used immunofluorescence and confocal microscopy to assess KLF protein levels in EA.hy926 cells treated with ox-LDL. As shown in Fig. 1A and B, KLF2, KLF3, KLF4, KLF6 and KLF7 (green) were all expressed in the nuclei, which are counter-stained with DAPI. KLF production was moderate without ox-LDL stimulation; however, incubation with $100 \mu \mathrm{g} / \mathrm{ml}$ ox-LDL for $24 \mathrm{~h}$ led to a significant decrease in KLF expression. The fluorescence intensity values of KLF2, KLF3, KLF4, KLF6 and KLF7 gene 
Table II. KLF expression in EA.hy926 cells after 24-h atorvastatin treatment.

\begin{tabular}{|c|c|c|c|c|}
\hline \multirow[b]{2}{*}{ Accession number } & \multirow[b]{2}{*}{ Gene name } & \multicolumn{2}{|c|}{$\begin{array}{l}\text { Average gray values in } \\
3 \text { biological repeats }\end{array}$} & \multirow{2}{*}{$\begin{array}{c}\begin{array}{c}\text { Fold change } \\
\text { (ranking) }\end{array} \\
\text { AT/DMSO }\end{array}$} \\
\hline & & DMSO & $\mathrm{AT}^{\mathrm{a}}$ & \\
\hline NM_016270 & Kruppel-like factor 2 (lung) (KLF2) & 4122.30 & 1504.64 & $2.74 \quad(72)$ \\
\hline NM_016531 & Kruppel-like factor 3 (basic) (KLF3) & 351.96 & 841.05 & $2.38(131)$ \\
\hline NM_004235 & Kruppel-like factor 4 (gut) (KLF4) & 101.20 & 433.28 & 4.27 (14) \\
\hline NM_001160124 & Kruppel-like factor 6 (KLF6) & 960.31 & 2044.95 & $2.13(225)$ \\
\hline NM_003709 & Kruppel-like factor 7 (KLF7) & 211.80 & 451.06 & $2.14(215)$ \\
\hline NM_001206 & Kruppel-like factor 9 (KLF9) & 49.81 & 104.61 & $2.03(276)$ \\
\hline NM_015995 & Kruppel-like factor 13 (KLF13) & 205.64 & 460.73 & $2.25(170)$ \\
\hline
\end{tabular}

${ }^{\mathrm{a}} \mathrm{AT}$, atorvastatin.

A

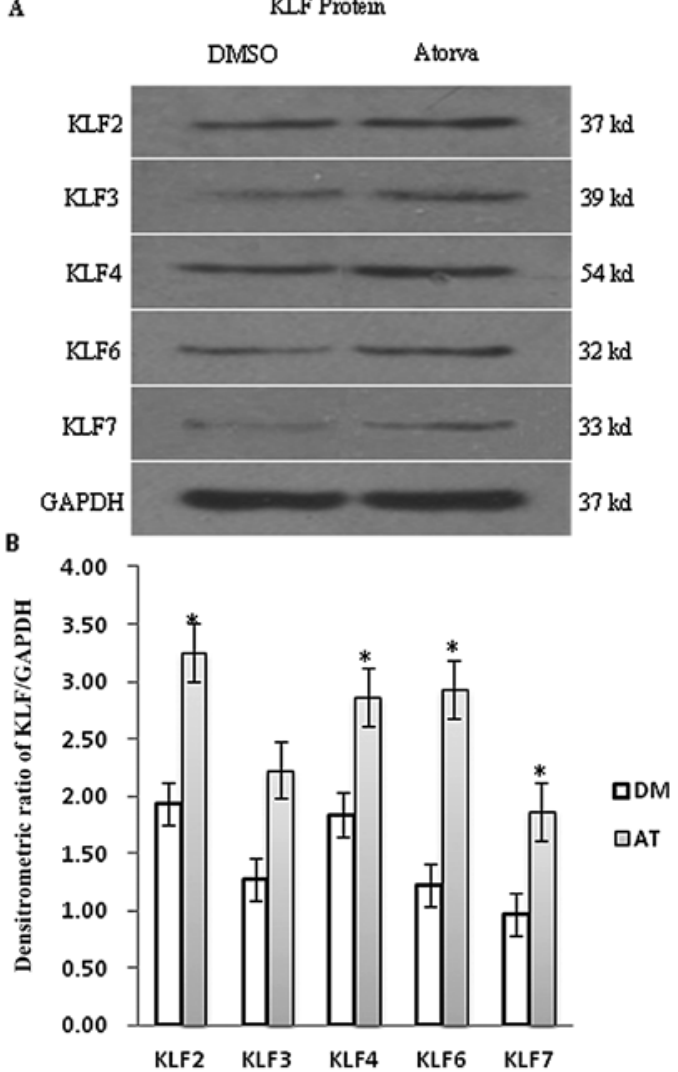

Figure 2. KLF protein was detected by western blot analysis. Incubation for $24 \mathrm{~h}$ with atorvastatin $(10 \mu \mathrm{M})$ increases KLF mRNA and KLF protein expression in EA.hy926 endothelial cells. (A) Representative western blot analyses of KLF2, KLF3, KLF4, KLF6, KLF7 and GAPDH expression. (B) Bar graphs show the mean gray values \pm SEM from analysis of DMSO group and atorvastatin group ( $\mathrm{n}=3$ each). ${ }^{*} \mathrm{P}<0.05$ AT vs. control group (DMSO). AT, atorvastatin.

expression before and after ox-LDL treatment were quantitatively analyzed and the results indicate a $>2$-fold decrease in KLF expression after ox-LDL treatment (Fig. 1C).

Atorvastatin upregulates KLF expression in quiescent EA.hy926 cells

DNA microarray analysis reveals that atorvastatin elevates the expression of KLF family members. To discover the molecular mechanism for the pleiotropic effects of atorvastatin, we conducted a cell-based microarray analysis to analyze the genome-wide transcriptional changes occurring in EA.hy926 cells after exposure to $10 \mu \mathrm{M}$ atorvastatin for $24 \mathrm{~h}$. Atorvastatin increased the expression of all KLF genes by $>2$-fold compared to DMSO. KLF4 showed the maximum change, with $>4$-fold upregulation (Table II).

Real-time PCR analysis validates the effect of atorvastatin on KLF gene expression. To validate the effect of atorvastatin on KLF gene expression, we used real-time PCR to measure mRNA levels in the EA.hy926 cells treated with atorvastatin for $24 \mathrm{~h}$. Under control (DMSO) conditions, KLF mRNA levels were low. Stimulation of EA.hy926 cells with 0.1-10 $\mu \mathrm{M}$ atorvastatin led to a concentration- and time-dependent increase in KLF mRNA expression that peaked $24 \mathrm{~h}$ after treatment with $10 \mu \mathrm{M}$ atorvastatin (data not shown). After $24 \mathrm{~h}$, the levels of KLF2, KLF4 and KLF13 mRNA were significantly higher in these cells than in the control cells $(\mathrm{P}<0.05)$ (as shown in Fig. 3C)

Atorvastatin promotes the expression of KLF protein in quiescent EA.hy926 cells. To confirm whether the change in KLF protein levels was consistent with PCR and DNA microarray results, KLF protein was analyzed and quantified by immunofluorescence and western blot analyses. As shown in Fig. 2, the expression of KLF2 and KLF4 protein in quiescent EA.hy924 cells was significantly higher than that of KLF3, KLF6 and KLF7. After incubation with $10 \mu \mathrm{M}$ atorvastatin for $24 \mathrm{~h}, \mathrm{KLF} 2, \mathrm{KLF} 3, \mathrm{KFL} 4, \mathrm{KLF} 6$ and KLF7 were all increased compared with the DMSO control, but protein levels were not altered to the same degree as the mRNA levels. The increase in KLF2 protein expression was <2-fold over that of the control and we found the same result by immunofluorescence analysis. Green fluorescence intensity values based on the binding intensity of the KLF antibodies were enhanced in the atorvastatin group compared to the DMSO group, but the change was $<2$-fold (Fig. 3A and B).

Atorvastatin counteracts ox-LDL-induced downregulation of KLF expression in EA.hy926 cells. As mentioned above, ox-LDL sharply decreased the levels of KLF protein, while atorvastatin upregulated the KLF expression at both the mRNA and protein levels. We further examined the effects of atorvastatin on ox-LDL-induced KLF expression by using 

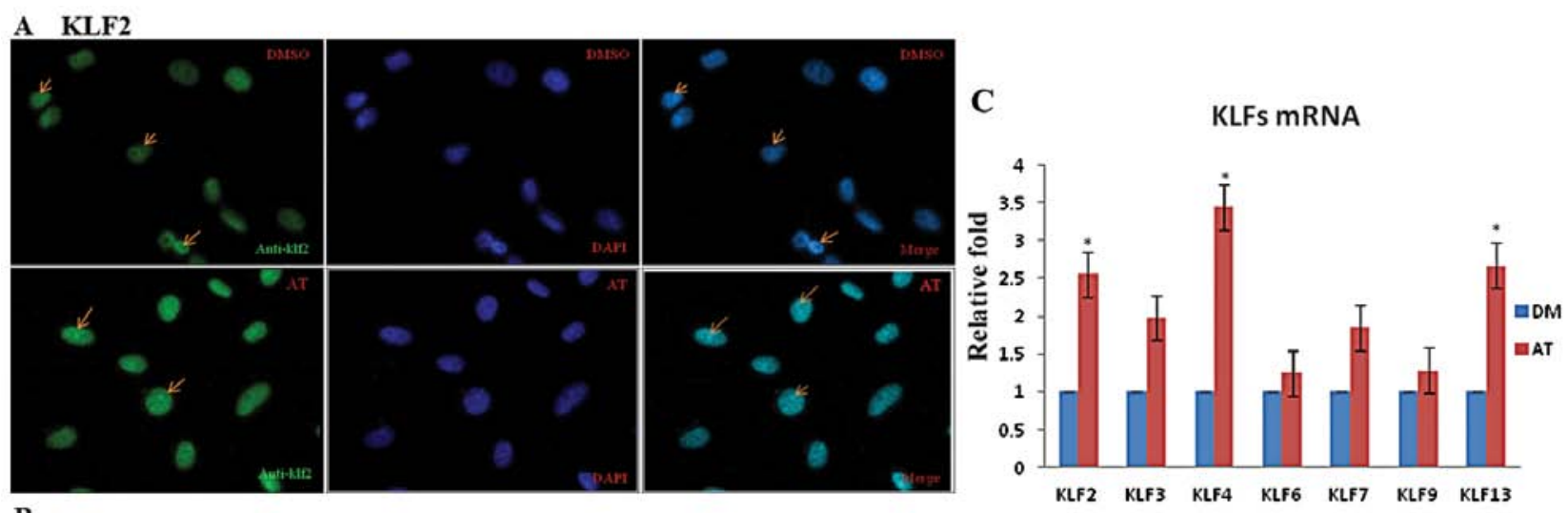

B

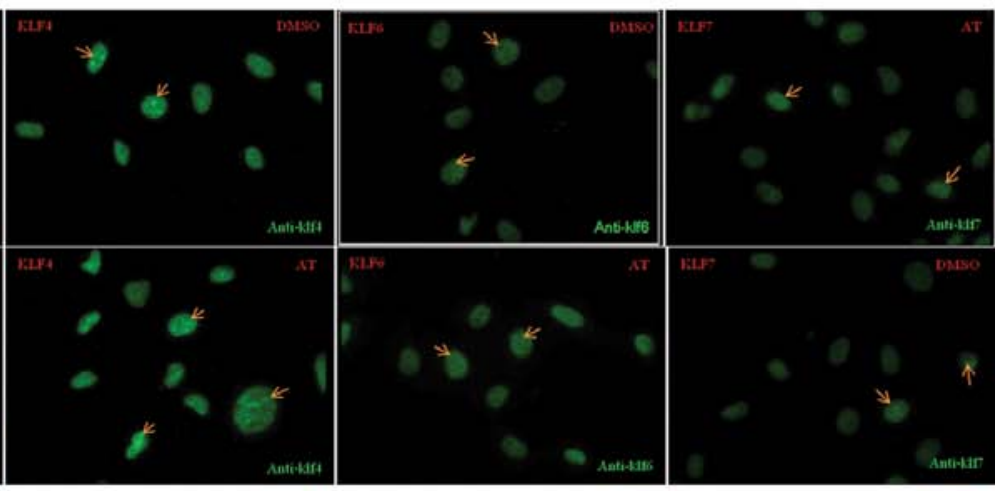

Figure 3. Relative quantification of KLF expression in EA.hy926 cells by real-time PCR and detection of KLF protein by immunofluorescence confocal microscopy. Incubation for $24 \mathrm{~h}$ with atorvastatin $(10 \mu \mathrm{M})$ increases KLF mRNA and KLF protein expression in EA.hy926 endothelial cells. (A and B) Representative immunofluorescence images of KLF protein treated with $10 \mu \mathrm{M}$ atorvastatin after $24 \mathrm{~h}$ compared with DMSO. Green represents anti-KLF2, KLF3, KLF4, KLF6 and KLF7 antibodies. Blue fluorescence represents DAPI nuclear staining. (C) Data are normalized to GAPDH and the KLF expression in the control (DMSO) group was set to 1 . Data are expressed as the mean $\pm \mathrm{SD}$ from 3 separate experiments. " $\mathrm{P}<0.05$ vs. control group; AT, atorvastatin.

immunofluorescence. In the nuclei of these cells, we observed a $>2$-fold decrease in KLF protein in the $100 \mu \mathrm{g} / \mathrm{ml}$ ox-LDL group compared to the DMSO control group (Fig. 1A and B), while the fluorescence intensity values of KLF2, KLF3, KLF4, KLF6 and KLF7 in ox-LDL + atorvastatin group were all elevated $>3$-fold over the ox-LDL alone group. These results demonstrate that atorvastatin counteracts the inhibitory effect induced by ox-LDL on KLF expression (Fig. 4).

\section{Discussion}

KLFs are members of the zinc finger family of transcription factors and previous studies have implicated these transcription factors as the key regulators of the endothelial pathways in vascular biology (18). KLF2 is the most widely studied KLF in endothelial cells and it is known as a 'molecular switch' that regulates the important aspects of vascular function and disease. KLF2 regulates endothelial thrombotic function by inducing the expression of potent anti-thrombotic and antiinflammatory genes such as eNOS, thrombomodulin and plasminogen activator inhibitor 1 (PAI-1). These results were further substantiated by siRNA knockdown experiments (8). In addition, KLF2 blocks endothelial cell activation by inhibiting interleukin (IL)-1 $\beta$ (19) and TNF- $\alpha$ (20), and inhibits angiogenesis by reducing endothelial cell proliferation and migration (21).

Both KLF4 and KLF10 are novel regulators of the inflammatory response $(22,23)$. KLF10 plays a central role in the anti-proliferative response via the TGF $\beta$-Smad pathway, which explains why KLF10 knockout mice develop cardiac hypertrophy (24). KLF4, which acts as a novel regulator of macrophage polarization, was robustly induced in M2 macrophages, whereas it was strongly reduced in M1 macrophages (25). KLF6 plays a key role in vascular development, remodeling and response to injury (12). KLF7 was recently reported to be a novel candidate for conferring susceptibility to type 2 diabetes (26). However, the underlying mechanisms responsible for endothelial cell injury with decreased KLF expression, remain to be elucidated.

In atherosclerosis, ox-LDL accumulates in the vessel walls and causes endothelial dysfunction, leading to the initiation and progression of atherosclerosis. However, the association between ox-LDL accumulation and KLF expression, as well as the effect of atorvastatin on ox-LDL-induced KLF expression, has not been determined. Therefore, we examined the effect of atorvastatin on ox-LDL-induced regulation of endothelial cell KLF expression.

Several studies have demonstrated that KLF2 is a novel nuclear mediator of the effects of statin in endothelial cells (27-30). In the present study, we found that ox-LDL can significantly decrease the mRNA expression of KLF2, KLF3, KLF4, KLF6, KLF7, KLF9 and KLF13. We further demonstrated that the downregulation of KLF expression by ox-LDL was counteracted by treatment with atorvastatin. These findings suggest that other KLFs, in addition to KLF2 and KLF4, play a role in the pathogenesis of atherosclerosis $(13,31)$. Downregulation 
A
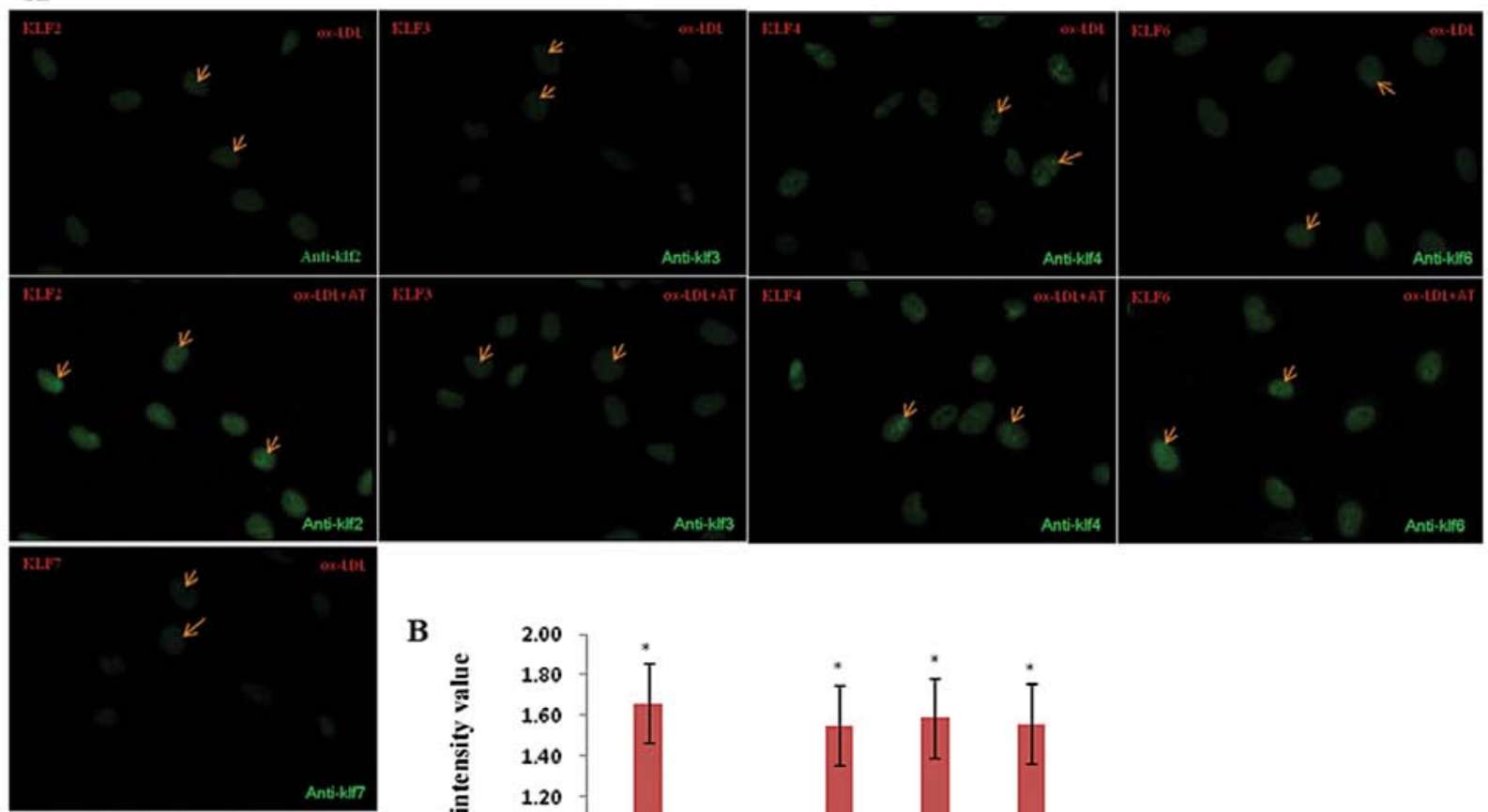

B

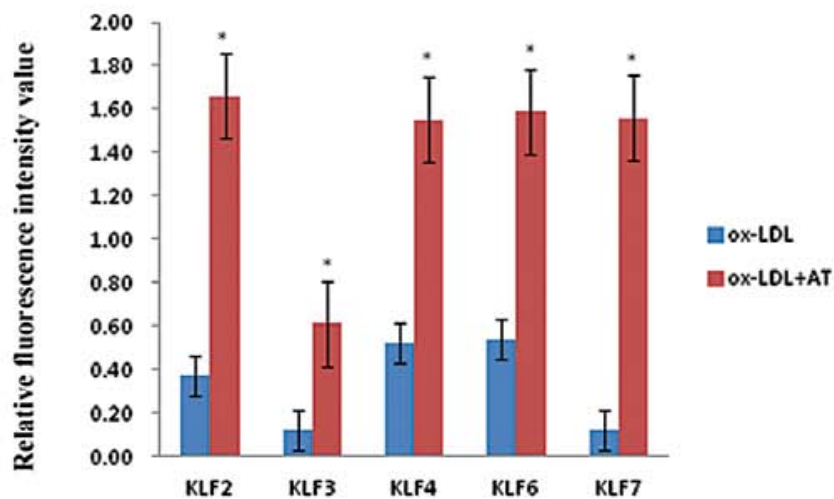

Figure 4. Atorvastatin upregulates ox-LDL-inhibited KLF protein expression as observed by immunofluorescence and confocal microscopy. (A) Representative immunofluorescence images are shown here. ox-LDL and ox-LDL + AT in the upper right corner of the pictures represent 2 groups: ox-LDL, cells were exposed to $100 \mu \mathrm{g} / \mathrm{ml}$ ox-LDL for $24 \mathrm{~h}$, and ox-LDL + AT, cells were exposed to $100 \mu \mathrm{g} / \mathrm{ml}$ ox-LDL $+10 \mu \mathrm{M}$ AT for $24 \mathrm{~h}$. (B) Bar graphs show the mean KLF fluorescent intensity values \pm SEM (n=3 each). $\mathrm{P}<0.05$ ox-LDL + AT group vs. ox-LDL group. AT, atorvastatin

of KLF expression by ox-LDL might be an effective target of atorvastatin in atherosclerosis. The signaling mechanisms orchestrating endothelial KLF2 expression as well as the expression of other KLFs in the vascular endothelium deserve further investigation.

Lectin-like oxidized low-density lipoprotein receptor-1 (LOX-1) is a major receptor for ox-LDL in the endothelial cells and is important for tumor growth, suggesting a molecular connection between atherogenesis and tumorigenesis (32-34). Recent studies show a positive correlation between increased serum ox-LDL levels and an increased risk of colon, breast, ovarian and esophageal cancers (35-37). Studies have revealed that KLF4, KLF9 and KLF10 have important tumor suppressor functions $(23,26,38-40)$. Based on our results that ox-LDL downregulates KLF expression, which would increase the risk of cancer, we hypothesized that KLFs were the targets of the pro-carcinogenic effect of ox-LDL and the anti-carcinogenic effect of atorvastatin.

Maintenance of the cellular participants of inflammatory reactions in a quiescent or inflammatory state is equally important. Therefore, we investigated these two states. Atorvastatin upregulated KLF expression in both the quiescent and ox-LDL-induced inflammatory states. This suggests that KLFs are involved in the control of cell proliferation and differentiation in normal as well as in pathological situations.
Although the mechanism of KLF reduction by ox-LDL is not well understood in EA.hy926 endothelial cells, our observations suggest that KLFs regulate the endothelial phenotype under both basal and inflammatory conditions. The evidence suggests that KLFs play an important role in endothelial dysfunction. Moreover, statin-induced upregulation of KLF expression may play an important role in the pharmacological and clinical effects of statins observed in cardiovascular diseases and carcinoma. Downregulation of KLFs in patient plasma might occur during the early stages of cardiovascular disease and cancer, and this may be correlated with disease severity, because ox-LDL levels are related to both atherosclerosis and an increased risk of cancer (33). Further clinical research is required to provide more information on the potential benefits of using statins to treat atherosclerotic diseases at an earlier stage.

\section{Acknowledgements}

This study was supported by the National Science and Technology Support Program (no. 2009BAI86B04).

\section{References}

1. Boon RA, Fledderus JO, Volger OL, et al: KLF2 suppresses TGF-beta signaling in endothelium through induction of Smad7 and inhibition of AP-1. Arterioscler Thromb Vasc Biol 27: 532-539, 2007. 
2. Mitra S, Goyal T and Mehta JL: Oxidized LDL, LOX-1 and atherosclerosis. Cardiovasc Drugs Ther 25: 419-429, 2011.

3. Ehara S, Ueda M, Naruko T, et al: Elevated levels of oxidized low density lipoprotein show a positive relationship with the severity of acute coronary syndromes. Circulation 103: 19551960, 2001.

4. Tsimikas S, Bergmark C, Beyer RW, et al: Temporal increases in plasma markers of oxidized low-density lipoprotein strongly reflect the presence of acute coronary syndromes. J Am Coll Cardiol 41: 360-370, 2003.

5. Cuadrado-Godia E, Ois A, Garcia-Ramallo E, et al: Biomarkers to predict clinical progression in small vessel disease strokes: prognostic role of albuminuria and oxidized LDL cholesterol. Atherosclerosis 219: 368-372, 2011.

6. Ryoo S, Bhunia A, Chang F, Shoukas A, Berkowitz DE and Romer LH: OxLDL-dependent activation of arginase II is dependent on the LOX-1 receptor and downstream RhoA signaling. Atherosclerosis 214: 279-287, 2011.

7. Mohty D, Pibarot P, Despres JP, et al: Association between plasma LDL particle size, valvular accumulation of oxidized LDL, and inflammation in patients with aortic stenosis. Arterioscler Thromb Vasc Biol 28: 187-193, 2008.

8. Oh GT, Choi JH, Hong JJ, et al: Dietary hematein ameliorates fatty streak lesions in the rabbit by the possible mechanism of reducing VCAM-1 and MCP-1 expression. Atherosclerosis 159: $17-26,2001$.

9. Zhao B, Luo X, Shi H and Ma D: Tissue factor pathway inhibitor-2 is downregulated by ox-LDL and inhibits ox-LDL induced vascular smooth muscle cells proliferation and migration. Thromb Res 128: 179-185, 2011.

10. Wang HH, Hsieh HL, Wu CY and Yang CM: Oxidized low-density lipoprotein-induced matrix metalloproteinase- 9 expression via PKC-delta/p42/p44 MAPK/Elk-1 cascade in brain astrocytes. Neurotox Res 17: 50-65, 2010.

11. Yu BL, Zhao SP and Huang XS: Oxidized low-density lipoprotein: a double-edged sword on atherosclerosis. Med Hypotheses 69: 553-556, 2007

12. Yu S, Wong SL, Lau CW, Huang Y and Yu CM: Oxidized LDL at low concentration promotes in-vitro angiogenesis and activates nitric oxide synthase through PI3K/Akt/eNOS pathway in human coronary artery endothelial cells. Biochem Biophys Res Commun 407: 44-48, 2011.

13. Yan FF, Liu YF, Liu Y and Zhao YX: KLF4: a novel target for the treatment of atherosclerosis. Med Hypotheses 70: 845-847, 2008

14. Villarreal G, Jr.,Zhang Y,Larman HB, Gracia-Sancho J, Koo A and Garcia-Cardena G: Defining the regulation of KLF4 expression and its downstream transcriptional targets in vascular endothelial cells. Biochem Biophys Res Commun 391: 984-989, 2010.

15. Lin Z, Kumar A, SenBanerjee S, et al: Kruppel-like factor 2 (KLF2) regulates endothelial thrombotic function. Circ Res 96 e48-e57, 2005

16. Azar RR, Badaoui G, Sarkis A, et al: Effect of ezetimibe/ atorvastatin combination on oxidized low density lipoprotein cholesterol in patients with coronary artery disease or coronary artery disease equivalent. Am J Cardiol 106: 193-197, 2010.

17. Ali F, Hamdulay SS, Kinderlerer AR, et al: Statin-mediated cytoprotection of human vascular endothelial cells: a role for Kruppel-like factor 2-dependent induction of heme oxygenase-1. J Thromb Haemost 5: 2537-2546, 2007.

18. Li H, Miao SB, Dong LH, et al: Clinicopathological correlation of Kruppel-like factor 5 and matrix metalloproteinase-9 expression and cartilage degeneration in human osteoarthritis. Pathol Res Pract 208: 9-14, 2011.

19. Racca AC, Camolotto SA, Ridano ME, Bocco JL, GentiRaimondi S and Panzetta-Dutari GM: Kruppel-like factor 6 expression changes during trophoblast syncytialization and transactivates sshCG and PSG placental genes. PLoS One 6 e22438, 2011.

20. Bialkowska AB, Crisp M, Bannister T, et al: Identification of small-molecule inhibitors of the colorectal cancer oncogene Kruppel-like factor 5 expression by ultrahigh-throughput screening. Mol Cancer Ther 10: 2043-2051, 2011.
21. Kee HJ, Kwon JS, Shin S, Ahn Y, Jeong MH and Kook H: Trichostatin A prevents neointimal hyperplasia via activation of Kruppel like factor 4. Vascul Pharmacol 55: 127-134, 2011.

22. Dang DT, Chen X, Feng J, Torbenson M, Dang LH and Yang VW: Overexpression of Kruppel-like factor 4 in the human colon cancer cell line RKO leads to reduced tumorigenecity. Oncogene 22: 3424-3430, 2003.

23. Mihailova A, Mikazane H, Klovins J and Nikitina-Zake L: Association of protein tyrosine phosphatase non-receptor 22 (PTPN22) rs2476601 and Kruppel-like factor 12 (KLF12) rs1324913 single nucleotide polymorphisms with rheumatoid arthritis in a Latvian population. Scand J Rheumatol 40: 491-492, 2011

24. Qin S, Liu M, Niu W and Zhang CL: Dysregulation of Kruppellike factor 4 during brain development leads to hydrocephalus in mice. Proc Natl Acad Sci USA 108: 21117-21121, 2011

25. Liao X, Sharma N, Kapadia F, et al: Kruppel-like factor 4 regulates macrophage polarization. J Clin Invest 121: 2736-2749, 2011

26. Kawamura Y, Tanaka Y, Kawamori R and Maeda S: Overexpression of Kruppel-like factor 7 regulates adipocytokine gene expressions in human adipocytes and inhibits glucoseinduced insulin secretion in pancreatic beta-cell line. Mol Endocrinol 20: 844-856, 2006.

27. McConnell BB, Kim SS, Yu K, et al: Kruppel-like factor 5 is important for maintenance of crypt architecture and barrier function in mouse intestine. Gastroenterology 141: 1302-1313, 2011.

28. Yori JL, Seachrist DD, Johnson E, et al: Kruppel-like factor 4 inhibits tumorigenic progression and metastasis in a mouse model of breast cancer. Neoplasia 13: 601-610, 2011.

29. Chang LK, Huang PH, Shen WT, Yang SH, Liu WJ and Lo CF: Role of Penaeus monodon Kruppel-like factor (PmKLF) in infection by white spot syndrome virus. Dev Comp Immunol 36: 121-129, 2012.

30. Parmar KM, Nambudiri V, Dai G, Larman HB, Gimbrone MA Jr and Garcia-Cardena G: Statins exert endothelial atheroprotective effects via the KLF2 transcription factor. J Biol Chem 280: 26714-26719, 2005.

31. SenBanerjee S, Lin Z, Atkins GB, et al: KLF2 Is a novel transcriptional regulator of endothelial proinflammatory activation. J Exp Med 199: 1305-1315, 2004.

32. Lu J, Mitra S, Wang X, Khaidakov M and Mehta JL: Oxidative stress and lectin-like ox-LDL-receptor LOX-1 in atherogenesis and tumorigenesis. Antioxid Redox Signal 15: 2301-2333, 2011.

33. Caiazzo M, Colucci-D'Amato L, Volpicelli F, et al: Kruppel-like factor 7 is required for olfactory bulb dopaminergic neuron development. Exp Cell Res 317: 464-473, 2011.

34. Lebson L, Gocke A, Rosenzweig J, et al: Cutting edge: The transcription factor Kruppel-like factor 4 regulates the differentiation of Th17 cells independently of RORgammat. J Immunol 185: 7161-7164, 2010.

35. Sivritas D, Becher MU, Ebrahimian T, et al: Antiproliferative effect of estrogen in vascular smooth muscle cells is mediated by Kruppel-like factor- 4 and manganese superoxide dismutase. Basic Res Cardiol 106: 563-575, 2011

36. Lu H, Wang X, Li T, et al: Identification of poly (ADP-ribose) polymerase-1 (PARP-1) as a novel Kruppel-like factor 8-interacting and -regulating protein. J Biol Chem 286: 20335-20344, 2011.

37. Moon JS, Kim HE, Koh E, et al: Kruppel-like factor 4 (KLF4) activates the transcription of the gene for the platelet isoform of phosphofructokinase (PFKP) in breast cancer. J Biol Chem 286: 23808-23816, 2011.

38. Guan H, Xie L, Leithauser F, et al: KLF4 is a tumor suppressor in B-cell non-Hodgkin lymphoma and in classic Hodgkin lymphoma. Blood 116: 1469-1478, 2010.

39. Yang Y, Goldstein BG, Chao HH and Katz JP: KLF4 and KLF5 regulate proliferation, apoptosis and invasion in esophageal cancer cells. Cancer Biol Ther 4: 1216-1221, 2005.

40. Bureau C, Hanoun N, Torrisani J, Vinel JP, Buscail L and Cordelier P: Expression and function of Kruppel like-factors (KLF) in carcinogenesis. Curr Genomics 10: 353-360, 2009. 